\title{
Multi criteria decision making methods for location selection of distribution centers
}

\author{
Romita Chakraborty ${ }^{a^{*}}$, Ankita Ray and Pranab K. Dan
}

Department of industrial engineering \&management, West Bengal University of Technology, Saltlake sector-1, Kolkata-700064, West Bengal, India

\section{H R O N I C L E}

Article history:

Received February 22013

Received in revised format

May 282013

Accepted June 162013

Available online

June 172013

Keywords:

Inflexible consumer demands

Facility location

Multi-criteria decision-making

(MCDM)

Ranking performance

REGIME \begin{abstract}
A B S T R A C T
In recent years, major challenges such as, increase in inflexible consumer demands and to improve the competitive advantage, it has become necessary for various industrial organizations all over the world to focus on strategies that will help them achieve cost reduction, continual quality improvement, increased customer satisfaction and on time delivery performance. As a result, selection of the most suitable and optimal facility location for a new organization or expansion of an existing location is one of the most important strategic issues, required to fulfill all of these above mentioned objectives. In order to sustain in the global competitive market of 21 st century, many industrial organizations have begun to concentrate on the proper selection of the plant site or best facility location. The best location is that which results in higher economic benefits through increased productivity and good distribution network. When a choice is to be made from among several alternative facility locations, it is necessary to compare their performance characteristics in a decisive way. As the facility location selection problem involves multiple conflicting criteria and a finite set of potential candidate alternatives, different multicriteria decision-making (MCDM) methods can be effectively applied to solve such type of problem. In this paper, four well known MCDM methods have been applied on a facility location selection problem and their relative ranking performances are compared. Because of disagreement in the ranks obtained by the four different MCDM methods a final ranking method based on REGIME has been proposed by the authors to facilitate the decision making process.
\end{abstract}

\section{Introduction}

A plant or facility is an entity that facilitates the execution of any job or process. Plant or facility location is the arrangement of process elements needed for the production of goods or delivery of services. It is an integrating phase of production system of an organization. The problem of selecting the best or most suitable facility location focuses on the evaluation of alternative locations by considering both qualitative and quantitative design criteria.

\footnotetext{
* Corresponding author.

E-mail: romita21@gmail.com (R. Chakraborty)

(C) 2013 Growing Science Ltd. All rights reserved.

doi: $10.5267 / \mathrm{j} . \mathrm{ijiec} .2013 .06 .006$
} 
Wang et al (2001) pointed out that in recent years, major challenges such as variable customer demand, short product life cycle and increasing worldwide competitions have aroused in the manufacturing industry. To overcome these challenges, an industry must be competent to provide world class quality products at lower costs than competitors and remain flexible in order to survive in the competitive business world (Yazdian \& Shahanaghi, 2011; Athawale \& Chakraborty, 2011). Ertay et al. (2006) considered that optimal design of physical location of a plant is an important issue in the early stage of system design. To achieve this goal, new manufacturing systems must be designed.

Facility location decisions are found to be extremely important for the long-term planning of the manufacturing organizations. Improper selection of location may result in inadequate qualified work force, unavailability of raw materials, insufficient transportation facility, increased operating expenses or even disastrous impact on the organization due to political and societal interference (Farhang Moghadam \& Seyedhosseini, 2010). Thus, it is very much necessary for the decision maker to select the appropriate location for a facility that will not only perform well, but also will be flexible enough to accommodate the necessary future changes. Selection of a proper location involves consideration of multiple feasible alternatives. It is also observed that the selection procedure involves several goals and it is often necessary to make compromise among the possible conflicting criteria. As a result, multicriteria decision-making (MCDM) methods are found to be an effective approach for solving these location selection problems.

The domain of FLP has been deeply explored by a number of researchers (Tompkins, \& Reed, 1976; Levary, \& Kalchik, 1985; Kusiak \& Heragu, 1987; Das, 1993; Meller \& Gau, 1996; Russell et al., 1999; Lin \& Sharp, 1999; Drira et al., 2007). Many researchers from the past have already applied various techniques to solve facility location selection problems. But most of those techniques were based on complex mathematical formulations, without considering the qualitative information regarding criteria values. Randhawa and West (1995) proposed a solution approach to facility location selection problems by integrating analytical and multi-criteria decision-making models.

Location theory was first introduced by Weber (see Brandeau \& Chiu, 1989) who considered the problem of locating a single warehouse in order to minimize the total travel distance between the warehouse and a set of spatially distributed costumers. In fact, he proposed a material index for selecting the location in which if this index is greater than one, the warehouse should be installed in the vicinity of the source of raw material; or otherwise, it should be close to the market. Isard (1956) reconsidered this work with the study of the industrial location, land use, and the related problems. Hotelling (1929) introduced another problem of locating two competing vendors along a straight line. Smithies (1941) and Stevens (1969) extended the Hotelling's problem later. Hakimi (1964) considered a general problem to locate one or more facilities on a network by minimizing the sum of the distances and the maximum distance between facilities and points on a network. Considerable research and theoretical interest in the location problem have been carried out after this seminal paper. Brown and Gibson (1972) and Buffa and Sarin (1987) proposed a facility location model for a multidimensional location problem based on critical factors, objective factors, and subjective factors. Fortenberry and Mitra (1986) presented a model for the location-allocation problems considering both qualitative and quantitative factors. Kahne (1975) considered 29 attributes and used a weighting model to determine the relative importance with uncertainty in attributes. Charnetski (1976) considered the case of selecting one of the three proposed sites for a modern air terminal with a large number of attributes.

In this paper, secondary data has been collected from a facility location selection problem by Shahanaghi and Yazdian (2010) comprising of multiple conflicting criteria and having a finite set of potential candidate alternatives and an attempt has been made to solve it with the help of five well known multi criteria decision making (MCDM) methodologies. Although a good amount of research work has been carried out in the past on solving facility layout selection problems using different mathematical approaches, specially employing multi-criteria decision-making (MCDM) methods, but 
very little attempt has been made to compare the relative performances of the MCDM methods while solving the decision-making problems. The main focus area of this paper is to compare the relative performances of the four well-known MCDM methods and to propose a final ranking model.

\section{Methodology}

In this section, a detailed illustration is presented regarding the steps that have been carried out to perform the comparative study on the ranking performance of various multi criteria decision making (MCDM) methods. These methods have been applied for solving a facility location selection problem and thereby drawing a conclusion justifying the satisfaction level of the below mentioned objectives. This approach mainly includes the following steps:

\subsection{Data collection defining objectives}

This is the first and the most important step of any multi criteria decision making problem. Before carrying out any type of research work, it is very essential for the researcher to have a transparent view of the objectives that his/her proposed work attempts to fulfill. In this paper, there are two main objectives that has been defined by the authors that include,

1. Application of four well known multi objective decision making methodologies for solving a facility location selection problem based on secondary data and then comparing their relative ranking performances

2. Proposing a final ranking method to facilitate the decision making process.

\subsection{Alternatives generation \& criteria fixation}

In this step, the layout alternatives are obtained from a simulation model. In addition; this step involves considering multiple conflicting criteria for solving the decision making problems. The criteria are mainly chosen by the decision maker depending on his/her judgments.

\subsection{Assigning criteria weights}

AHP developed by Saaty (1980) provides a method to decompose the complex problem into a hierarchy of sub problems which can be evaluated and handled more easily and rationally. AHP also helps in evaluating the weights associated with various criteria in order to judge the importance of each and every criterion.

\subsection{Ranking location alternatives}

In this step five different MCDM methods have been applied to solve the facility location selection problem for the purpose of ranking the layout alternatives. These methods include,

1. Grey Relational Analysis (GRA)

2. Multi Objective Optimization On The Basis OF Ratio Analysis (MOORA)

3. Elimination Of Choice Translating Reality (ELECTRE II)

4. Operational Competitiveness Rating Analysis (OCRA)

\subsection{Comparative analysis}

To determine the applicability and suitability of the four above cited MCDM methods while solving the facility location selection problem, their relative ranking performances are compared using the following measures: 
a) Spearman's rank correlation coefficient,

b) Kendall's rank correlation coefficient,

c) Agreement between the top three ranked alternatives

\subsection{Decision making}

After ranking the alternatives according to relative significance of each alternative, final ranking method based on REGIME has been proposed by the authors to facilitate the decision making process.

\section{Multi- criteria decision- making methods}

Multiple criterion decision making (MCDM) refers to making decisions in the presence of multiple, usually conflicting criteria. In strategic planning, various MCDM methods are presently being applied which can also be effectively used to select the most appropriate facility layout for a given industrial organization. But the large number of available MCDM methods, of varying complexity and possibly solutions, confuses the potential decision maker. Hence, the decision maker also faces the problem of selecting the most appropriate MCDM method from among several feasible alternatives. Any MCDM problem can be represented by a matrix $(X)$ consisting of $m$ alternatives and $n$ criteria.

$$
X=\left[\begin{array}{ccc}
x_{11} & \cdots & x_{1 n} \\
\vdots & \ddots & \vdots \\
x_{m 1} & \cdots & x_{m n}
\end{array}\right]_{m \times n}
$$

Where, $x_{i j}$ is the performance measure of $i^{\text {th }}$ alternative on $j^{\text {th }}$ criterion. In MCDM methods, it is also required to determine the priority weight $\left(w_{j}\right)$ of each criterion such that the sum of weights for all the criteria equals to one. These priority weights can be determined using AHP or entropy method.

In this paper, in order to compare the ranking performances of different MCDM methods while solving a facility location selection problem, the following MCDM methods are used,

\subsection{GREY RELATIONAL ANALYSIS (GRA)}

The procedural steps of GRA method are described as follows:

Step 1: Generate the grey relation using an appropriate normalization procedure.

Step 2: Define the reference sequence.

For $j^{t h}$ criterion of ith alternative, if the value $x_{i j}$ is equal to 1 , or nearer to 1 than the value of the other alternatives, the performance of $i^{\text {th }}$ alternative is the best one for that $j^{\text {th }}$ criterion. Therefore, an alternative will be the best choice if all of its performance values are closest to or equal to 1 . The reference alternative is defined as $x_{0}$.

Step 3: Calculate the grey relational coefficient.

Grey relational coefficient is used to determine how close $x_{i j}$ is to $x_{0 j}$. The larger the grey relational coefficient, the closer $x_{i j}$ and $x_{0 j}$ are. It is calculated using the following equation:

$\gamma\left(x_{0 j}, x_{i j}\right)=\frac{\Delta_{\min }+\zeta \Delta_{\max }}{\Delta_{i j}+\zeta \Delta_{\max }}$ 
where, $\gamma\left(x_{i j},, x_{0 j}\right)$ is the grey relational coefficient between $x_{i j}$ and $, x_{0 j}, \Delta_{i j}=\left|x_{0 j}-x_{i j}\right|, \Delta \min$ is the minimum value of $\Delta_{i j}$ and $\Delta_{\max }$ is the maximum value of $\Delta_{i j}$. $\zeta$ is the distinguishing coefficient which ranges from 0 to 1 and generally, is taken as 0.5 .

Step 4: Compute the grey relational grade $(\mathrm{GRG})$ value as follows:

GRG between $x_{i}$ and $x_{o}=\sum_{j=1}^{n} w_{j} \gamma\left(x_{0 j}, x_{i j}\right)$

The GRG represents the level of correlation between the reference sequence and the comparability sequence. If the comparability sequence for an alternative gets the highest GRG value with the reference sequence, it means that the comparability sequence is most similar to the reference sequence and that alternative is the best choice.

\subsection{Multi objective optimization on the basis of ratio analysis (MOORA)}

Multi-objective optimization (or programming), also known as multi-criteria or multi-attribute optimization, is the process of simultaneously optimizing two or more conflicting attributes (objectives) subject to certain constraints. The MOORA method, first introduced by Brauers (2004) is such a multiobjective optimization technique that can be successfully applied to solve various types of complex decision making problems in the manufacturing environment.

The procedural steps of MOORA method are described as follows:

Step 1: Brauers et al. (2008) concluded that for this denominator, the best choice is the square root of the sum of squares of each alternative per attribute. This ratio can be expressed as below:

$$
X_{i j}^{*}=\frac{X_{i j}}{\sqrt{\sum_{i=1}^{m} X_{i j}^{2}}}, j=1, \cdots, n
$$

where, $X_{i j}$ is a dimensionless number which belongs to the interval $[0,1]$ representing the normalized performance of $i^{\text {th }}$ alternative on $j^{\text {th }}$ attribute.

Step 2: For multi-objective optimization, these normalized performances are added in case of maximization (for beneficial attributes) and subtracted in case of minimization (for non-beneficial attributes). Then the optimization problem becomes:

$$
Y_{i}=\sum_{j=1}^{g} X_{i j}^{*}-\sum_{j=g+1}^{n} X_{i j}^{*}
$$

where, $g$ is the number of attributes to be maximized, $(n-g)$ is the number of attributes to be minimized, and $\mathrm{y}_{\mathrm{i}}$ is the normalized assessment value of $i^{\text {th }}$ alternative with respect to all the attributes. When the attribute weights are taken into consideration, Eq. (7) becomes as follows:

$Y_{i}=\sum_{j=1}^{g} W_{j} X_{i j}^{*}-\sum_{j=g+1}^{n} W_{j} X_{i j}^{*}, j=1, \cdots, n$

where, $W_{j}$ is the weight of $\mathrm{j}^{\text {th }}$ attribute, which can be determined applying analytic hierarchy process (AHP) or entropy method.

Step 3: The $Y_{i}$ value can be positive or negative depending of the totals of its maxima (beneficial attributes) and minima (non beneficial attributes) in the decision matrix. An ordinal ranking 
of $Y_{i}$ shows the final preference. Thus, the best alternative has the highest $Y_{i}$ value, while the worst alternative has the lowest $y_{i}$ value.

\subsection{Elimination of Choice Translating Reality (ELECTRE II)}

The ELECTRE method developed by Roy and Vincke (1981) is based on multi-attribute utility theory with the intention to improve efficiency without affecting the outcome while considering less information. The aim of this outranking method is to find all alternatives that dominate other alternatives while they cannot be dominated by any other alternative. In ELECTRE method, every pair of the alternatives $A_{i}$ and $A_{k}$ is assigned a concordance index, $c(i, k)$ which can be expressed as below:

$c(i, k)=\sum_{g_{j}(i) \geq g_{j}(k)} w_{j}, \mathrm{i}, \mathrm{k}=1, \ldots, \mathrm{m} ;(\mathrm{i} \neq \mathrm{k})$

where, $g_{j}(i)$ and $g_{j}(k)$ are the normalized measures of performance of ith and kth alternative respectively with respect to $j^{\text {th }}$ criterion. Thus, for an ordered pair of alternatives $\left(A_{i}, A_{k}\right)$, the concordance index, $c(i, k)$ is the sum of all the weights for those criteria where the performance score of $A_{i}$ is at least as that of A $\mathrm{k} \mathrm{A}$ discordance index, $d(i, k)$ is also calculated as given below:

$d(i, k)=\left\{\begin{array}{c}0 \text { if } g_{j}(i) \geq g_{j}(k) \\ \max \left\{g_{j}(k)-g_{j}(i)\right\} /{ }_{j=1}^{n} \max \left\{g_{j}(k)-g_{j}(i)\right\} i, k=1,2, \ldots m ; i \neq k\end{array}\right.$

Once these two indices are determined, an outranking relation $\mathrm{S}$ can be defined as:

$A_{i} S A_{k}$ if and only if $c(i, k) \geq c^{\prime}$ and $d(i, k) \geq d^{\prime}$ where, c' and d' are the threshold values as set by the decision maker.

The steps for ELECTRE method are described as below:

Step 1: Obtain the normalized values of all the criteria.

Step 2: Construct the outranking relations by following the concordance and discordance definitions, and develop a graph representing the dominance relations among the alternatives. In this graph, if alternative $A_{i}$ outranks alternative $A_{k}$, then a directed arc exists from $A_{i}$ to $A_{k}$.

Step 3: Obtain a minimum dominating subset by using the minimum concordance and maximum discordance indices.

Step 4: If the subset has a single element or is small enough to apply value judgment, select the final decision. Otherwise, steps 2 to 4 are repeated until a single element or small subset exists.

Step 5: If a full ranking of the alternatives is required, apply an extension of ELECTRE (ELECTRE II) method.

Another two indices are calculated as follows:

Pure discordance index $\left(D_{i}\right)=\sum_{k=1}^{m} c(i, k)-\sum_{k=1}^{m} c(k, i)(i \neq k)$
Pure discordance index $\left(D_{i}\right)=\sum_{k=1}^{m} d(i, k)-\sum_{k=1}^{m} d(k, i)(i \neq k)$ 
a) Once these two indices are estimated, obtain two rankings on the basis of these indices.

b) Determine an average ranking from the two rankings, as obtained in step 5(a).

c) Select that alternative which has the best average rank.

\subsection{Operational Competitiveness Rating Analysis (OCRA)}

OCRA method was developed to measure the relative performances of a set of production units (PUs), where resources are consumed to create value-added outputs. The preference ratings of the alternatives in OCRA method reflect the decision maker's preferences for the criteria.

The general OCRA procedure is described as below:

Step 1: Compute the preference ratings with respect to the non beneficial criteria.

In this step, OCRA method is only concerned with the scores that various alternatives receive for the input criteria without considering the scores received for the beneficial criteria. The aggregate performance of $i^{\text {th }}$ alternative with respect to all the input criteria is calculated using the following equation:

$\bar{I}_{i}=\sum_{j=1}^{m} W_{j} \frac{\max \left(X_{i j}\right)-X_{i j}}{\min \left(X_{i j}\right)}$,

where, $\bar{I}_{i}$ is the measure of the relative performance of ith alternative and $X_{j}^{i}$ is the performance score of $i^{\text {th }}$ alternative with respect to $j^{\text {th }}$ input criterion.

Step 2: Calculate the linear preference rating for the input criteria.

$\overline{\bar{I}}_{i}=\bar{I}_{i}-\min \left(\bar{I}_{i}\right)$

This linear scaling is done to assign a zero rating to the least preferable alternative. $\overline{\bar{I}}_{i}$ represents the aggregate preference rating for ith alternative with respect to the input criteria.

Step 3: Compute the preference ratings with respect to the beneficial criteria. The aggregate performance for $i^{\text {th }}$ alternative on all the beneficial or output criteria is measured using the following expression:

$\bar{O}_{i}=\sum_{n=1}^{h} W_{j}\left[\frac{X_{i j}-\min X_{i j}}{\min X_{i j}}\right]$

Where, $h=1,2, \ldots, j$ indicates the number of beneficial attributes or output criteria and $W j$ is calibration constant or weight importance of hth output criteria. The higher an alternative's score for an output criterion, the higher is the preference for that alternative.

Step 4: Calculate the linear preference rating for the output criteria using the following equation:

$\overline{\bar{O}}_{i}=\bar{O}_{i}-\min \left(\bar{O}_{i}\right)$

Step 5: Compute the overall preference ratings. The overall preference rating for each alternative is calculated by scaling the sum $\left(\overline{\bar{I}}_{i}+\overline{\bar{O}}_{i}\right)$ so that the least preferable alternative receives a rating of zero. The overall preference rating $\left(P_{i}\right)$ is calculated as follows:

$P_{i}=\overline{\bar{I}}_{i}+\overline{\bar{O}}_{i}$ 
The alternatives are ranked according to the values of the overall preference rating. The alternative with the highest overall performance rating receives the first rank.

\subsection{Final Ranking Method (REGIME)}

The REGIME method was described by Hinloopen et al. (1983) and Hinloopen and Nijkamp (1986). This method is nothing but an ordinal generalization of pairwise comparison methods such as concordance analysis. The procedural steps of REGIME is described as follows,

Step 1: Computing the concordance $C_{i l}$ using the following equation:

$C_{i l}=\sum_{j \in \widehat{c_{l l}}} \pi_{j}$

$\widehat{C_{l l}}=$ the set of concordance which reflects the set of attributes for which $a_{i}$ is at least as good as $a_{l}$, where $a_{i}$ and $a_{l} \in A . \pi_{j}$ is the weight of the attribute $g_{j} \in F$.

Step 2: Construction of regime matrix by pair-wise comparison of alternatives in the multi-criteria evaluation table. For every criterion, it is examined whether $a$ has a better rank than $b$, then on the corresponding place in the regime matrix the number +1 is noted, while if $b$ is a better position than $a$, then -1 will be placed in the regime matrix.

Step 3: Next, for each criterion $g_{j}$ an indicator $C_{i l, j}$ for each pair of alternatives $\left(a_{i}, a_{l}\right)$ can be defined as,

$C_{i l, j} C_{i l}=\left\{\begin{array}{c}+1 \text { if } r_{i j}<r_{l j} \\ 0 \text { if } r_{i j}=r_{l j} \\ -1 \text { if } r_{i j}>r_{l j}\end{array}\right.$

$r_{i j}\left(r_{l j}\right)=$ the rank of the alternative $a_{i}\left(a_{l}\right)$ with respect to the attribute $g_{j}$

The elements of the regime matrix are called regimes and they are used to determine the rank order of alternatives.

The concordance index, for the alternative $a_{i}$ is given by:

$C_{i l}=\sum_{j} \pi_{j} C_{i l, j}$

Step 4: Construction of a pair-wise comparison matrix $V_{i l}$, defined as,

$V_{i l}= \begin{cases}+1 & \text { if } C_{i l}>0 \\ -1 & \text { if } C_{i l}<0\end{cases}$

This matrix consists of elements equal to 1 or -1 , and zeroes on main diagonal. A final ranking can be achieved on the basis of matrix $V_{i l}$. The alternative that has maximum number of positive elements i.e., +1 in the matrix $V_{i l}$ is considered as the best alternative.

\section{Case study}

In this paper, the layout design proposed by Shahanaghi and Yazdian (2010) on the area of "Facility location and distribution decisions in supply chains with fleet sizing considering both tangible and intangible criteria" is taken into consideration. They had employed a two-phase approach to deal with 
this problem. In the first phase, the decision maker scores and ranks all potential DC locations with regard to a set of criteria, and in the second phase through a multi-objective mixed integer programming (MOMIP) model final location and distribution decisions, which incorporates selection of transportation modes and their associated loads, are made. They have used AHP to determine the relative importance of each criterion and TOPSIS to do the final ranking. This example takes into account five facility location selection criteria and four alternative distribution centers (DC). The five criteria considered in this problem are, Fire history (F); Access to infrastructures (I); Reliability in operations (R); Closeness to market (M); Expert personnel availability (P); Earthquake possibility (E).

The same example is considered here to demonstrate the applicability and effectiveness of five other well known MCDM methods.

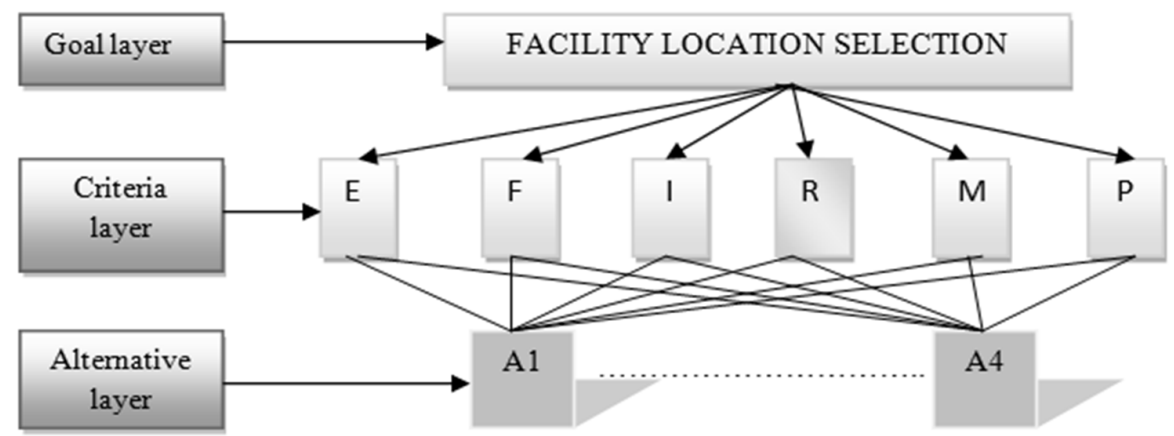

Fig. 1. Hierarchy structure of the facility location problem

The decision matrix for rank calculation is as follows,

Table 1

Decision matrix for rank evaluation

\begin{tabular}{|c|c|c|c|c|c|c|}
\hline Alternatives & $\mathrm{E}$ & $\mathrm{F}$ & $\mathrm{I}$ & $\mathrm{R}$ & $\mathrm{M}$ & $\mathrm{P}$ \\
\hline Criteria weights & 0.255 & 0.394 & 0.047 & 0.107 & 0.034 & 0.164 \\
\hline $\mathrm{A} 1$ & 3 & 1 & 7 & 3 & 7 & 5 \\
\hline A2 & 1 & 5 & 5 & 5 & 9 & 3 \\
\hline A3 & 7 & 1 & 5 & 5 & 3 & 7 \\
\hline A4 & 5 & 3 & 9 & 7 & 7 & 5 \\
\hline
\end{tabular}

\section{Result analysis}

The mentioned facility location selection problem is solved using the following MCDM methods,

\subsection{GRA method}

In this method, after normalizing the original decision matrix, the grey relational coefficients are calculated using eq. (1), where the value of the distinguishing coefficient $(\zeta)$ is taken as 1 . Next, the corresponding GRG values are determined, as shown in Table 2. After arranging the GRG values in descending order, the ranking of the alternative facility locations are obtained as follows,

Table 2

Grey relational grades

\begin{tabular}{ccc}
\hline Alternatives & GRG & Rank \\
\hline A1 & 0.8049 & 1 \\
A2 & 0.6628 & 4 \\
A3 & 0.7973 & 2 \\
A4 & 0.7044 & 3 \\
\hline
\end{tabular}




\subsection{MOORA method}

After normalizing the decision matrix using eq. (3), the performances $Y i$ of each of the alternatives are calculated using eq. (5) and thereby the facility location alternatives are ranked in descending order, as follows,

Table 3

Performance scores using MOORA method

\begin{tabular}{ccc}
\hline Alternatives & $\boldsymbol{Y i}$ & Rank \\
\hline A1 & 0.0026 & 1 \\
A2 & -0.2175 & 4 \\
A3 & -0.07336 & 2 \\
A4 & -0.1362 & 3 \\
\hline
\end{tabular}

\subsection{ELECTRE II method}

To solve this problem using ELECTRE II method, the original decision matrix is first normalized. In order to obtain the full ranking of the alternative locations, the corresponding pure concordance and discordance indices are computed, as given in Table 4.Then the final ranking is obtained by calculating an average rank as shown below,

Table 4

Ranking of facility locations using ELECTRE II method

\begin{tabular}{ccccccc}
\hline Alternatives & $\boldsymbol{C} \boldsymbol{i}$ & Initial rank & $\boldsymbol{D} \boldsymbol{i}$ & Initial rank & Average rank & Final rank \\
\hline $\mathrm{A} 1$ & 0.769 & 1 & 0.0669 & 2 & 1.5 & 1 \\
$\mathrm{~A} 2$ & -0.901 & 4 & 0.5751 & 3 & 3.5 & 4 \\
$\mathrm{~A} 3$ & 0.319 & 2 & 0.8334 & 4 & 3 & 3 \\
$\mathrm{~A} 4$ & -0.817 & 3 & -1.4754 & 1 & 2 & 2 \\
\hline
\end{tabular}

\subsection{OCRA method}

The overall preference rating $(P i)$ is calculated using Eq. (13) and the alternatives are ranked accordingly by descending order as shown in Table 5 . The facility location alternative having the highest value of $P_{i}$ is considered as the best choice.

Table 5

Ranking of facility locations using OCRA method

\begin{tabular}{ccccccc}
\hline Alternatives & $\overline{\mathrm{I}}_{\mathbf{i}}$ & $\overline{\boldsymbol{O}}_{\boldsymbol{i}}$ & $\overline{\overline{\boldsymbol{I}}}_{\boldsymbol{i}}$ & $\overline{\boldsymbol{\boldsymbol { O }}}_{\boldsymbol{i}}$ & $\boldsymbol{P}_{\boldsymbol{i}}$ & Final rank \\
\hline $\mathrm{A} 1$ & 2.596 & 0.1731 & 1.298 & 0.0338 & 1.1365 & 1 \\
$\mathrm{~A} 2$ & 1.53 & 0.1393 & 0.232 & 0 & 0.0367 & 3 \\
$\mathrm{~A} 3$ & 1.576 & 0.29 & 0.278 & 0.1507 & 0.2334 & 2 \\
$\mathrm{~A} 4$ & 1.298 & 0.3346 & 0 & 0.1953 & 0 & 4 \\
\hline
\end{tabular}

The ranking results obtained by applying four different MCDM methods on the facility location selection problem are listed in Table 6.

Table 6

Comparative analysis of ranking of facility locations using MCDM methods

\begin{tabular}{ccccc}
\hline Alternatives & GRA & MOORA & ELECTRE II & OCRA \\
\hline A1 & 1 & 1 & 1 & 1 \\
A2 & 4 & 4 & 4 & 3 \\
A3 & 2 & 2 & 3 & 2 \\
A4 & 3 & 3 & 2 & 4 \\
\hline
\end{tabular}


From the above table, it is evident that GRA and MOORA are providing similar set of rankings for the alternatives. However, the rankings obtained by ELECTRE II and OCRA vary from each other as well as the other two methods. Thus, it can be concluded that there is no perfect agreement between the ranks obtained by the four different MCDM methods.

\section{Comparative analysis}

Next, to determine the applicability and suitability of the above-cited MCDM methods to solve this facility location selection problem, their relative ranking performance are compared using the following measures:

a) Spearman's rank correlation coefficient,

b) Kendall's rank correlation coefficient,

c) Agreement between the top three ranked alternatives

Using Spearman's rank correlation coefficient $\left(r_{s}\right)$ value, the similarity between two sets of rankings can be measured. Usually, its value lies between -1 and +1 , where the value of +1 denotes a perfect match between two rank orderings. It is calculated as,

$r_{s}=1-\frac{6 \sum d_{i}^{2}}{n\left(n^{2}-1\right)}$

where, $d_{i}=$ difference between ranks of alternatives by two methods, $n=$ no. of observations.

The Kendall rank correlation coefficient, commonly referred to as Kendall's tau $(\tau)$ coefficient, is a technique applied to measure the relationship between two measured quantities. Specifically, it is a measure of rank correlation, i.e., the similarity of the orderings of the data when ranked by each of the quantities. Normally, its value lies between -1 and +1 , where the value of +1 denotes a perfect match between two rank orderings. It is calculated as,

$\mathrm{T}=\frac{\text { (no. of concordant pairs })-(\text { no. of discordant pairs })}{0.5 n(n-1)}$,

where, $n=$ total no. of pair combinations

As, the decision maker may be sometimes interested to select the best facility location as the single choice, another test is performed based on the agreement between the top three ranked location alternatives. Here, a result of $(1,2,3)$ means the first, second and third ranks match; $(1,2, *)$ means the first and second ranks match; $(1, *, *)$ means only the first ranks match; and $(*, *, *)$ means no match.

\section{Table 8:}

Performance test results

\begin{tabular}{ccccc}
\hline MCDM methods & GRA & MOORA & ELECTRE II & OCRA \\
\hline GRA & - & 1 & 0.8 & 0.8 \\
MOORA & & $(1,2,3)$ & $(1,2, *)$ & $\left(1, *,^{*}\right)$ \\
ELECTRE II & & - & 0.8 & 0.8 \\
& & & $(1,2, *)$ & $(1, *, 3)$ \\
OCRA & & & - & 0.4 \\
& & & & $\left(1, *,^{*}\right)$ \\
\hline
\end{tabular}


The value of Kendall's coefficient of concordance (T) is computed as -0.4 , which suggests that there is an almost no perfect agreement between the considered MCDM methods. Therefore, another ranking method is proposed by the authors that combines the ranking results of the four MCDM methods. This ranking method is very similar to REGIME method. The only difference is in place of criteria used in REGIME, we are considering the four MCDM methods as the criteria. The main objective behind doing so is to obtain a composite final rank that combines the ranking results of all the other methods.

Table 9

Impact matrix

\begin{tabular}{ccccc}
\hline Alternatives & GRA & MOORA & ELECTRE II & OCRA \\
\hline A1 & 1 & 1 & 1 & 1 \\
A2 & 4 & 4 & 4 & 3 \\
A3 & 2 & 2 & 3 & 2 \\
A4 & 3 & 3 & 2 & 4 \\
\hline
\end{tabular}

Table 10

Regime matrix

\begin{tabular}{lcccc}
\hline Alternatives & GRA & MOORA & ELECTRE II & OCRA \\
\hline A1,A2 & 1 & 1 & 1 & 1 \\
A1,A3 & 1 & 1 & 1 & 1 \\
A1,A4 & 1 & 1 & 1 & 1 \\
A2,A1 & -1 & -1 & -1 & -1 \\
A2,A3 & -1 & -1 & -1 & -1 \\
A2,A4 & -1 & -1 & -1 & 1 \\
A3,A1 & -1 & -1 & -1 & -1 \\
A3,A2 & 1 & 1 & 1 & 1 \\
A3,A4 & 1 & 1 & -1 & 1 \\
A4,A1 & -1 & -1 & -1 & -1 \\
A4,A2 & 1 & 1 & 1 & -1 \\
A4,A3 & -1 & -1 & 1 & -1 \\
\hline
\end{tabular}

In this case the authors have assigned equal weightage to the criteria for the calculation of the concordance index $C_{i l}$. Therefore for each criterion, $\mathbb{T}_{j}$ is taken as $1 / 4$. The paired comparison matrix $V_{i l}$ for composite rank calculation is shown in Table 11. The final ranking obtained by this method is noted in Table 12.

Table 11

Paired comparison matrix

\begin{tabular}{ccccc}
\hline Alternatives & A1 & A2 & A3 & A4 \\
\hline A1 & 0 & 1 & 1 & 1 \\
A2 & -1 & 0 & -1 & -1 \\
A3 & -1 & 1 & 0 & 1 \\
A4 & -1 & 1 & -1 & 0 \\
\hline
\end{tabular}

Table 12

Final rank of alternatives

\begin{tabular}{ccc}
\hline Alternatives & Total no. of positive elements $(+1)$ & Final rank \\
\hline A1 & 3 & 1 \\
A2 & 0 & 4 \\
A3 & 2 & 2 \\
A4 & 1 & 3 \\
\hline
\end{tabular}




\section{Conclusion}

This paper investigated the ranking performance of four most well-known MCDM methods while solving a facility location selection problem. It has been observed that the rankings were significantly influenced by the choice of the MCDM methods employed. The discrepancy that appears between the rankings obtained by various MCDM methods is due to the difference in their mathematical modelling while solving a decision problem. Thus, it becomes very difficult for the decision maker to decide that which ranking method is best suited for the problem. Thus, in this paper a final ranking model based on REGIME method has been proposed by the authors. Since this model combines the ranking results obtained by four different methods instead of depending on the results of just one or two MCDM methods, therefore, this model can be considered more reliable for the decision problems.

The future scope of this paper may include comparing the relative performance of the other newly developed MCDM methods, like QUALIFLEX, MAPPAC, ARAS, COPRAS (complex proportional assessment), LINMAP (linear programming technique for multi-dimensional analysis of preference) and NAIADE (novel approach to imprecise assessment and decision environments) while solving the facility location selection problems.

Another dimension can be given to this facility location problem by integrating it with various soft computing techniques like genetic algorithm, artificial neural networks, meta-heuristics, fuzzy goal programming etc.

\section{References}

Athawale, V. M., \& Chakraborty, S. (2011). A comparative study on the ranking performance of some multi-criteria decision-making methods for industrial robot selection. International Journal of Industrial Engineering Computations, 2(4), 831-850.

Brandeau, M. L., \& Chiu, S. S. (1989). An overview of representative problems in location Research. Management Science, 35(6), 645-674.

Brauers, W.K.M. (2004). Optimization methods for a stakeholder society. A revolution in economic thinking by multiobjective optimization. Kluwer Academic Publishers, Boston.

Brauers, W.K.M., Zavadskas, E.K., Peldschus, F., \& Turskis, Z. (2008). Multiobjective decisionmaking for road design. Transport, 23, 183-193.

Brown, P. A., \& Gibson, D. F. (1972). A quantified model for facility site selection: An application to a multi facility location problem. AIIE Transactions, Vol. 4, 1-10.

Buffa, E. S., \& Sarin, R. K. (1987). Modern production and operations management. 8th ed., John Wiley and Sons, New York.

Charnetski, J. R. (1976). Multiple criteria decision making with partial information: A site selection problem. Space Location-Regional Development, Pion, London, 51-62.

Das, S.K. (1993). A facility layout method for flexible manufacturing systems. International Journal of Production Research, 12(3), 279-297.

Drira, A., Pierreval, H. \& Hajri-Gabouj, S. (2007). Facility layout problems: A survey. Annual Reviews in Control, 31(2), 255-267.

Ertay, T., Ruan, D. \& Tuzkaya, U.R. (2006). Integrating data envelopment analysis and analytic hierarchy for the facility layout design in manufacturing systems. Information Sciences, 176(3), 237262.

Farhang Moghadam, B., \& Seyedhosseini, S. M. (2010). A particle swarm approach to solve vehicle routing problem with uncertain demand: A drug distribution case study. International Journal of Industrial Engineering Computations, 1(1), 55-64.

Fortenberry, J. C., \& Mitra, A., (1986). A multiple criteria approach to the location-allocation problem. Computer and Industrial Engineering, 10(1), 77- 87. 
Hakimi, S. L. (1964). Optimal locations of switching centers and the absolute centers and medians of a graph, Operations Research, 12, 450-459.

Hinloopen, E., Nijkamp, P., \& Rietveld, P. (2004). Integration of ordinal and cardinal information in multi-criteria ranking with imperfect compensation.European Journal of Operational Research, 158(2), 317-338.

Hinloopen, E., Nijkamp, P., \& Rietveld, P. (1983). Qualitative discrete multiple criteria choice models in regional planning. Regional Science and Urban Economics, 13, 77-102.

Hotelling, H. (1929). Stability in competition. Economic Journal, 39, 41-57.

Isard, W. (1956). Location and space economy. Technology Press, MIT, Cambridge, MA.

Kahne, S. (1975). A procedure for optimizing development decisions. Automatica, 11(3), 261- 269.

Kusiak, A. \& Heragu, S.S. (1987). The facilities layout problem. European Journal of Operational Research, 29(3), 229-251.

Levary, R.R. \& Kalchik, S. (1985). Facilities layout - a survey of solution procedures. Computers \& Industrial Engineering, 9(2), 141-148.

Lin, L.C. \& Sharp, G.P. (1999). Quantitative and qualitative indices for the plant layout problem. European Journal of Operational Research, 116(1), 118-138.

Meller, R.D. \& Gau, K.Y. (1996). The facility layout problem: recent and emerging trends and evaluation perspectives. Journal of Manufacturing Systems, 15(5), 351-66.

Rao, R.V.( 2007). Decision Making in the Manufacturing Environment using Graph Theory and Fuzzy Multiple Attribute Decision Making Methods. Springer-Verlag, London.

Randhawa S.U., \& West T.M. (1995). An integrated approach to facility location problem. Computers and Industrial Engineering, 29, 261-265.

Roy, B., \& Vincke, P. (1981). Multi-criteria analysis: Survey and new directions. European Journal of Operational Research, 8, 207-218.

Russell, D.M., Venkat, N. \& Pamela, H.V. (1999). Optimal facility layout design. Operations Research Letters, 23(3), 117-127.

Saaty, T.L. (1980). The analytic hierarchy process. New York: McGraw-Hill.

Shahanaghi, K., \& Yazdian, S.A. (2010). Facility location and distribution decisions in supply chains with fleet sizing considering both tangible and intangible criteria. International Journal of Management Science and Engineering Management, 5(4), 302-308.

Sheskin, D.J. (2004). Handbook of parametric and nonparametric statistical procedures. Chapman and Hall/CRC.

Smithies, A., (1941). Optimum location in spatial competition. Journal of Political Economy, 49, 432492.

Stevens, B. H. (1969). An application of game theory to a problem in location strategy. Papers of Regional Science Association, 7, 85-111.

Tompkins, J.A. \& Reed, J.R. (1976). An applied model for the facilities design problem. International Journal of Production Research, 14(2), 583-595.

Wang, T.Y., Wu, K.B. \& Liu, Y.W. (2001). A simulated annealing algorithm for facility layout problems under variable demand in cellular manufacturing systems. Computers in Industry, 46(2), 181-188.

Yazdian, S. A., \& Shahanaghi, K. (2011). A multi-objective possibilistic programming approach for locating distribution centers and allocating customers' demands in supply chains. International Journal of Industrial Engineering Computations, 2(1), 193-202. 